\title{
Topical Issue on many particle spectroscopy of atoms, molecules, clusters and surfaces editorial
}

\author{
Károly Tőkési ${ }^{1, a}$, Béla Paripás², Gábor Pszota² , and Andrey V. Solov’yov,4,b \\ 1 Institute for Nuclear Research (Atomki), Debrecen, Hungary \\ 2 Institute of Physics, University of Miskolc, Miskolc-Egyetemváros 3515, Hungary \\ 3 MBN Research Center, Altenhöferallee 3, 60438 Frankfurt am Main, Germany \\ 4 A.F. Ioffe Physical-Technical Institute, Polytekhnicheskaya ul. 26,, St. Petersburg, Russia 194021
}

(C) The Author(s), under exclusive licence to EDP Sciences, SIF and Springer-Verlag GmbH Germany, part of Springer Nature 2021

\begin{abstract}
Many particle spectroscopy is a subject of continued interest to many experimental and theoretical groups worldwide. It is based on the coincidence spectroscopy of minimum two particles coming from the same elementary process. It is a very powerful tool for studying not just atoms and molecules but also more extended electronic systems such as clusters and surfaces. Due to the large variety of its applications, it is really an interdisciplinary research field. This Topical Issue presents a state-of-the-art description of current research activities in the field of many particle spectroscopy. The contributions to this Issue represent original research results on both experimental and theoretical studies, involving the interaction of various projectiles, like photons, electrons, ions with atoms, molecules, clusters and surfaces.
\end{abstract}

\section{Introduction}

In the early decades of atomic physics, one could detect only one out of the particles (no matter how many there were) that were produced in an elementary atomic process, or at least there was no information about multiple detections. The coincidence technique that was introduced a little more than $50 \mathrm{y}$ ago represented a qualitative jump in this area as well. Using this method, one can simultaneously measure the parameters of two particles that were produced in the same elementary atomic process. Later triple or even quadruple, coincidences became measurable.

At the beginning, both the primary particle starting the atomic physics process and the two particles in the final state, detected in coincidence, were mainly electrons. This (e,2e) technique dominated the field of interest even twenty years ago [1], but publications with ion or photon projectiles also appeared. During the last two decades, the fast development of synchrotrons and lasers caused significant changes, primarily in the nature of the projectiles. Publications with photon projectiles gradually became the majority $[2,3]$, and then, they became dominant $[4,5]$.

The objective of this Topical Issue is to assess the state of the art in the current understanding of a variety of basic phenomena in the charged particle dynamics in atoms, molecules, clusters and at surfaces such as:

\footnotetext{
a e-mail: tokesi@atomki.hu

b e-mail: solovyov@mbnresearch.com (corresponding author)
}

collision induced physical, chemical and biological reactions, ultrafast dynamics, charge-exchange processes, collective as well as single-particle excitation and ionization, electron-electron correlation effects in atoms and in solids, excitation and single and multiple ionization of various targets, energy loss, scattering and channelling of primary particles, electron and photon emission processes.

\section{Experimental advances in electron and ion impact processes}

The experimental study of atoms by means of electron projectiles is a traditional field of interest. Such a work is reported by Borovik et al. [6], where the excitation-autoionization (EA) cross section for the $5 p^{6}$ subshell in $\mathrm{Ba}$ atoms was determined in an incident electron energy range from $15.61 \mathrm{eV}$ up to $600 \mathrm{eV}$. The data were obtained by measuring the total intensity of ejected-electron spectra arising from the decay of the $5 p^{5} n_{1} l_{1} n_{2} l_{2} n_{3} l_{3}$ autoionizing states. They found that the energy dependence of the cross section is characterized by the presence of a strong resonance structure in the near-threshold energy region. Genevriez et al. [7] reported on the experimental determination of absolute, total cross sections for electron-impact double ionization of helium in its metastable $1 s 2 s^{3} \mathrm{~S}$ state and of the helium negative ion $\mathrm{He}-\left(1 s 2 s 2 p^{4} \mathrm{P}\right)$. Cross sections were measured for impact energies ranging from threshold to $1000 \mathrm{eV}$ using the animated crossed beams tech- 
nique. The $\mathrm{He}^{-}$beam was produced by double charge capture of fast $\mathrm{He}+$ ions in sodium vapour and the pure, fast beam of metastable helium by subsequent photodetachment of the He-beam. It was found that the cross section for double ionization of $\mathrm{He}-\left(1 s 2 s 2 p^{4} \mathrm{P}\right)$ is large compared to other negative ions, as a consequence of its very small binding energy and its hollow-core electronic configuration. They also identified two different ionization regimes in the Bethe plot of the cross section. At low energies, direct double ionization of the two outer electrons dominates, while at higher energies, ionization of the 1 s core electron followed by autoionization becomes more important.

Tókési et al. [8] studied the state-to-state (exchange) interference of the autoionizing resonances of helium in $(\mathrm{e}, 2 \mathrm{e})$ experiments. These studies were disturbed by the coincidence events caused by direct ionization [9], so their decrease is desirable. For this reason, to mimic the experimental observation, the authors performed fourbody classical trajectory Monte Carlo calculations. The calculations were done, following the same experiments, for $93.15 \mathrm{eV}$ primary energy, where the exchange interference of the $2 \mathrm{~s}^{2}\left({ }^{1} \mathrm{~S}\right)$ and $2 \mathrm{p}^{2}\left({ }^{1} \mathrm{D}\right)$ autoionizing states of helium is expected. According to the CTMC results, the authors found that the forward-backward asymmetry of the electrons coming from direct ionization is greater in the coincidence experiments with the applied geometry than in non-coincidence experiments. This observation was in agreement with the experimental findings.

Dampc and co-workers [10] used an electron-impact mass spectroscopy to investigate ionization and cationic fragmentation of the pyridazine (1,2 diazine), $\mathrm{C}_{4} \mathrm{H}_{4} \mathrm{~N}_{2}$, molecules in the gas phase. The mass spectra were measured, and the observed mass peaks were assigned to the corresponding cations. They found that at higher electron-impact energy, electrons from valence orbitals of the cations, with higher binding energies, may be removed. This destabilizes the cations and may initiate dissociation (dissociative ionization), dipolar dissociation and also Coulomb explosion.

The combined experimental and theoretical works were also performed to study the absolute single and double electron loss (stripping) and single and double electron capture cross sections for $0.2-1.2 \mathrm{MeV} \mathrm{O}^{+}$projectiles colliding with water molecules [11]. In order to mimic the experimental observation, three-, four- and five-body classical trajectory Monte Carlo calculations were carried out. They found that the calculated cross sections are in agreement with the experimental data [12]. Santos et al. [13] have shown both theoretical and experimental results for the Auger electron spectra after the chlorine $2 \mathrm{p}$ excitation and ionization in the gas phase $\mathrm{CH}_{2} \mathrm{Cl}_{2}$ and $\mathrm{CD}_{2} \mathrm{Cl}_{2}$ molecules. They also presented a series of high level ab initio quantum mechanical calculations at multi-configurational selfconsistent field (MCSCF) and multi-reference configuration interaction (MRCI) levels of theory to compute the $\mathrm{Cl}$ L2;3VV Auger lines. Calculations were compared with the corresponding experimental photo-excited and photo-ionized spectra. Maljkovic and co-workers investigated the elastic scattering of electrons from triethyl phosphate molecules experimentally and theoretically in the energy range from $50 \mathrm{eV}$ to $250 \mathrm{eV}$ [14]. The experimental set-up based on a crossed beam technique comprising of an electron gun, a single capillary gas needle and a detection system with a channeltron was used to measure differential cross sections. The absolute scale for the cross sections was obtained by relative-flow method using argon gas as a reference. For the interpretation of the measured data, the partial expansion method was used. Although the calculation procedure was crude, they found excellent agreement between the shapes of measured and calculated data.

\section{Theoretical advances}

\subsection{Photon-induced processes}

The interest towards the interpretation of photoninduced processes is continuously growing. Numerous calculations were performed for the photon projectileatom target combination. The photo-ionization of rubidium in strong infrared laser fields based on ab initio calculations was investigated [15]. The bound and the continuum states were described with Slater orbitals and Coulomb wave packets, respectively. The bound state spectra were calculated with the variational method. The authors found that it reproduced the experimental data within a few percent accuracy. The effects of the shape and the parameters of the pulse to the photoionization probabilities and the energy spectrum of the ionised electrons were shown. We note that these calculations may provide a valuable contribution at the design of laser and plasma-based novel accelerators, the CERN AWAKE experiment.

Lopez and Arbo have elucidated the relevance of the Coulomb potential in the interference structures observed in the momentum distribution of atomic ionization using different theoretical models [16]. They found that by increasing the importance of the Coulomb potential in the phase of the semi-classical approximations, the separation among the interference fringes grows. They also have shown that not only the role of the Coulomb potential is crucial for the formation of the interference structures, but also it needs to be properly accounted for in the theory. Novikovskiy et al. [17] presented a detailed theoretical analysis of the $1 \mathrm{~s}$ photoionization of neon. It was found that the most significant many-electron correlation in computing photoionization of inner shells is the rearrangement of the outer shells caused by the inner vacancy. Further noticeable effects are: (1) the polarization of the ion core by the outgoing photoelectron and (2) the coherent effect of double excitation/ionization.

Three articles [18-20] deal with the different photoionization processes of argon. Among them, Lagutin and co-workers studied the two-photon ionization of the $3 p$ shell of Ar in the above-threshold ionization (ATI) exciting-photon energy region [18]. The ab initio calculations were performed using perturbation the- 
ory and the correlation function technique. The latter enables to perform a summation over the complete set of intermediate states emerging in the transition amplitude. Generalized two-photon ionization cross sections were calculated taking into account many-electron correlations. The calculations demonstrated that the twophoton ionization of Ar at the $3 p^{4}$ threshold is almost entirely a collective process. The decisive contribution in the above-threshold two-photon $3 p$-ionization at the exciting-photon energies corresponding to the $3 p$ $>\varepsilon d$ giant resonance comes from the Auger-like manyelectron correlations of $\varepsilon^{\prime} d \varepsilon^{\prime \prime} d-3 p \varepsilon f$ type. Gryzlova presented theoretical studies of two-photon sequential double ionization of Ar for photon energies in the region of Rydberg autoionizing states $3 p^{4}\left({ }^{1} D\right) n l$ of the ion $\mathrm{Ar}^{+}$ overlapping with the particle-hole autoionizing states $3 s 3 p^{6} n p$ of neutral Ar [19]. They found that the atomic and ionic autoionizing states lead to sharp variations of the angular correlation function between the two outgoing electrons, as well as in the angular distributions of the first and the second emitted electrons. A strong influence of the second step ionization on the first ionization step was demonstrated. In the third contribution, Orimo et al. presented comparisons between quantum and classical calculations for above threshold ionization of an Argon atom interacting with a strong laser pulse [20]. The time-dependent complete-activespace self-consistent field (TD-CASSCF) method and the classical trajectory Monte Carlo (CTMC) method were used for the cases of quantum and classical calculations, respectively. The authors discussed the differences between the results obtained by the TD-CASSCF and the CTMC simulations.

For the photo-ionization of various molecules, the investigations were carried out to study the effect of the strong laser field [21], the interchannel mixing [22] and the angular distribution parameters [23]. ShvetsovShilovsk et al. published a pioneer work regarding the semi-classical approach for molecular target [21]. They extended the semi-classical two-step model for strong-field ionization that describes quantum interference and accounts for the Coulomb potential beyond the semi-classical perturbation theory to the hydrogen molecule. In the simplest case of the molecule oriented along the polarization direction of a linearly polarized laser field, they predicted significant deviations of the two-dimensional photoelectron momentum distributions and the energy spectra from the case of atomic hydrogen. Specifically, for the hydrogen molecule the electron energy spectrum falls off slower with increasing energy, and the holographic interference fringes are more pronounced than for the hydrogen atom at the same parameters of the laser pulse. Rearrangement of the electron shells accompanying inner-shell photoionization of the $\mathrm{CH}_{4}$ molecule was also studied theoretically [22]. Novikovskiy and co-workers calculated the K-shell photo-ionization cross sections and the corresponding photoelectron angular distribution parameters in different approximations using the single centre method. They found a strong mixing of the partial ionization channels in the vicinity of the $1 s$ ion- ization threshold, caused by the non-spherical part of the molecular potential, which results in a qualitative difference between the electronic rearrangement effects in molecules, as compared to atoms. Novikovskiy et al. also published theoretical results for photo-ionization cross sections and photoelectron angular distribution parameters of the closed-shell $\mathrm{CH}_{4}, \mathrm{NH}_{3}, \mathrm{H}_{2} \mathrm{O}$ and $\mathrm{CO}$ molecules [23]. For the neon-like molecules, calculations were performed in two approximations: in the spherical single-orbital and in the non-spherical many-orbital approaches. In the case of $1 a_{1}(1 s)$ shells of $\mathrm{CH}_{4}, \mathrm{NH}_{3}$ and $\mathrm{H}_{2} \mathrm{O}$, the photo-absorption cross sections obtained in the simplified FC SO approximation fortuitously coincide with the results of the more accurate RAC SC calculations.

After exchanging the target, the photoelectron spectra simulations were performed for nanotips as well. The photoelectron trajectories emitted from silver nanoparticles were calculated using the classical trajectory Monte Carlo method, where the image force towards the surface was taken into account [24]. It was shown that the code developed for these special simulations describes the experimental findings reasonably well. Significant effect of the image acceleration to the calculated electron spectra was observed. They found that the calculated energy distributions of photoelectrons are in agreement with the recent experiments.

\subsection{Electron-induced processes}

The laser-assisted (e,2e) collision [25] is a sort transition between the photon and electron projectiles. For the normal $(\mathrm{e}, 2 \mathrm{e})$ processes - which dominated the program in 2000-nowadays they only perform a small amount of calculations. Zaytsev et al. studied the laser-assisted fast $(e ; 2 e)$ collisions on atomic hydrogen both in perturbative and in non-perturbative regimes of laser radiation. The authors calculated the multi-photon triple differential cross sections in asymmetric coplanar kinematics employing the first Born approximation in the projectile-target interaction. They have shown that in both radiation regimes, their method exhibits high efficiency for different net numbers of photons exchanged between the colliding system and the laser field.

For electron projectiles, calculations for double ionization of helium [26] and electron Monte Carlo simulations in solids were also presented [27,28]. Zaytsev et al. presented fully differential cross sections for the double ionization of Helium by fast electron impact [26]. Ionization amplitudes were obtained by solving in the entire space, to first order, the corresponding three-body-driven Coulomb Schrödinger equation. The comparison with two other ab initio first-order calculations (CCC and GSF) shows that the obtained FDCS shapes were very similar. For certain geometrical situations, however, the authors found a substantial magnitude difference when the two electrons are ejected with $4 \mathrm{eV}$ each. Being slower for this lower energy case, the two electrons interact more (stronger correlation). The authors attributed the magnitude difference to the 
difficult asymptotic electron-electron Coulomb phase: while it does not appear naturally with coupled products of one-electron basis functions (CCC and GSF), within our approach, such phase is built in from the outset. The energy loss functions (ELFs) of three transition metals ( $\mathrm{Cr}$, Co and $\mathrm{Pd}$ ) have been derived from the reflection electron energy loss spectroscopy spectrum with a theoretical analysis of the measured data [27]. The ELFs were presented in a wide photon energy region (0-200 eV) with very high accuracy. The accuracy of the calculations was verified by sum rules. The electron inelastic mean free paths (IMFPs) of Cr, Co and $\mathrm{Pd}$ have also been shown based on the obtained ELFs by adopting a dielectric response theory. During the simulation, both the single-pole approximation and full Penn algorithm for the calculation of IMFPs were applied. Gong and co-workers presented an analysis of electron incident angle-dependent reflected electron energy loss spectroscopy spectra of silver by using a Monte Carlo simulation method, with which the separated contributions from surface and bulk excitations can be identified [28]. The simulations were performed at several different incident electron energies and with various incident angles with respect to the surface normal. They found that the surface plasmon excitation plays a dominant role in the loss peak at around 3.7 and $7.5 \mathrm{eV}$.

\subsection{Particles, antiparticles, exotic particles}

The influence of the sign of charge of the projectiles for the cross sections for light projectiles [29] as well as for muons and pions [30] was also presented. Positron- and electron-impact ionization of the $1 b_{1}$ and $3 a_{1}$ orbitals of $\mathrm{H}_{2} \mathrm{O}$ was studied by CDW-EIS model at an impact energy of $81 \mathrm{eV}$ [29]. Fully differential cross sections were calculated and contrasted after a proper average procedure over molecular orientations. Double binary peaks were observed for positron-impact and coplanar geometries, while only single binary peaks are exhibited for electron-impact ionization. For the denominated perpendicular planes, similar structures are observed, with an enhancement or decrease in the binary and recoil lobes. Forward electron emission was analysed identifying the electron capture to the continuum structure for positron impact. A high narrow peak and a huge dip for positron and electron impact are, respectively, observed, structures already seen in previous ionization studies involving both light and heavy projectiles. The K-shell ionization cross sections by positively and negatively charged muons and pions have been published at low energies with the CTMC and the ECPSSR [30]. The obtained results are in good agreement with each other and also with the CC calculations for muons on $\mathrm{Cu}$. The ECPSSR cross sections by negative projectiles are larger than the PWBA, and the values for positive projectiles are in the opposite direction. The reasons can be ascribed to the binding-energy effect of the target electron and the Coulomb-deflection effect of the projectile. In the energy region considered here, the latter effect is dominant. At the same projectile velocity, the cross section for pions is almost the same as that for muons, but the deviation from the PWBA is slightly larger for muons. This is explained as the difference in the Coulomb-deflection effect.

Following the work with muon projectiles, the energy shifts and relative radiative rates of electronic $\mathrm{K}_{\alpha} x$ rays were presented for light muonic atoms with various states of the muon by Mukoyama [31]. The calculations were based on some simplified assumptions, but $n$ dependence of the muonic orbital on the energy shifts and radiative rates was obtained. The author found that the $l$ dependence is small. It was assumed that electron vacancies created during muon cascade are quickly refilled. However, for light muonic atoms this assumption may not be valid. The calculations indicated that the number of $2 p$ electrons at the moment of electronic K-x-ray emission plays an important role in energy shifts and relative radiation rates. The knowledge of electron vacancy distribution in cascade processes is essential to obtain more accurate information from analysis of electronic X-rays for light muonic atoms. For this purpose, we may need further experimental and theoretical studies on light muonic atoms.

\subsection{Collisions with heavy particles}

For the case of heavy projectiles, calculations were presented for targets of helium [32] and lithium atoms [33], as well as for water molecules [34], and neutral atoms and hydrogen molecules [35] collisions. Differential cross sections for charge-exchange and single and double electronic emission in collisions of protons with He-atoms at intermediate impact energies were theoretically evaluated by the classical trajectory Monte Carlo methods [32]. The models incorporate momentum-dependent terms to the Hamiltonian in order to avoid the classical autoionization of He. The theoretical results for single capture and single ionization were compared to available experimental data. The role of the electronelectron correlation effects in double ionization processes was analysed by inspecting the angular and energetic dependence of the electronic emission spectra at different impact energies. Bondarev et al. considered the single ionization of lithium atoms in collisions with $p$ and $\mathrm{O}^{8+}$ projectiles [33]. Doubly differential cross sections for ionization were reported within a relativistic non-perturbative approach and compared with the recent measurements. Otranto and co-workers studied theoretically, using the classical trajectory Monte Carlo method, the electron production following single and multiple ionization of water by bare ion impact [34]. The obtained results may provide a benchmark against experimental data collected in different laboratories and other theoretical data obtained by means of distorted wave methods. During the simulations, ten electrons in the molecule were explicitly considered, in contrast to previous formulations based on a single active electron description of the target. The authors presented a simple scaling of the cross sections. The 
collisional radiative models used in the modelling of beam emission spectroscopy diagnostics neglect atomatom collisions because of a lack of sufficiently detailed atomic data. Filling this scantiness, Asztalos and coworkers presented 4-body classical trajectory Monte Carlo simulations to calculate the cross sections for various channels in collisions between $\mathrm{H}+\mathrm{H}_{2}$ and $\mathrm{Li}+\mathrm{H}_{2}$ for a wide range of projectile energies [35]. Based on the calculated cross sections, a simplified version of the collisional radiative model has been derived. The authors have shown that the model is suitable to obtain the beam attenuation in neutral gases outside of the confined plasma region. A strong density dependence has been found for each beam species.

\subsection{Molecules and attophysics}

Finally, in this issue the works with hydrocarbon molecules [36] and the generation of attosecond laser pulses [37] were presented. Zhao et al. [36] studied theoretically the Auger effect after inner-valence ionization of several unsaturated and saturated cyclic and linear hydrocarbon molecules. These prototype molecules were chosen such that the effects of the different characteristics of aromaticity ( $\pi$ electrons, conjugation, cyclic geometry) on the Auger decay can be investigated separately. The authors have shown that among these molecules, the ones having $\pi$ electrons can undergo Auger decay after inner-valence ionization. Furthermore, the results reported suggest that conjugation allows for several open Auger decay channels, while aromaticity limits the range of the latter. Last but not least new theoretical and numerical results were presented regarding isolated attosecond XUV soft X-ray pulses that can be generated by Thomson backscattering of a high-intensity single-cycle near infrared laser pulse on a suitable nanobunch of $\mathrm{MeV}$ electrons [37]. The theoretical investigations of Hack et al. have shown that the Thomson backscattering of a NIR laser pulse on a suitable relativistic electron nanobunch is a promising source of an isolated attosecond XUV soft $X$-ray pulse, having advantageous features. Based on the analysis of the coherence factor, the authors derived a simple formula for the cut-off frequency of the collective radiation spectrum, which could also be useful in designing the corresponding experiments.

\section{Concluding remarks}

Many particle spectroscopy has continued its significant progress during the previous years. The objective of this issue was to assess the state of the art in the current understanding of a variety of basic phenomena: in the charged particle dynamics in atoms, molecules, clusters and at surfaces such as collisioninduced physical, chemical and biological reactions, ultrafast dynamics, charge-exchange processes, collective as well as single-particle excitation and ionization, electron-electron correlation effects in atoms and in solids, excitation and single and multiple ionization of various targets, energy loss, scattering and channelling of primary particles and electron and photon emission processes. However, a significant number of interesting problems are still unresolved and many interesting fields of physics are waiting for investigations. It is still open to investigate the interaction between many electronic systems either in ion-atom, ion-molecule, ioncluster or ion-surface collisions. As a new feature, in the last years, attoscience applying lasers came to the fore [38-43]. Attosecond physics is a new and rapidly developing field driven by recent advances in laser technology. Attosecond science holds the promise to observe and to control the optical-field-driven electronics working at clock speeds six orders of magnitude faster than current conventional electronics. Combining this extremely small time-scale with nanodimensional materials gives us fairly new techniques for new discoveries, especially applying the technique of the many particle spectroscopy.

All the old and new questions like physical and chemical phenomena induced by the interaction of particles (singly up to highly charged ions, atoms and clusters, electrons and photons) with atoms, molecules, clusters and surface and the bulk of solids and also liquids remain the key topics of the many particle spectroscopy. The present Topical Issue, based on the works reported at the International Conference on Many Particle Spectroscopy of Atoms, Molecules, Clusters and Surfaces (MPS-2018), summarized and highlighted the current understanding of a variety of basic phenomena in the charged particle dynamics in atoms, molecules, clusters and at surfaces such as collision induced physical, chemical and biological reactions, ultrafast dynamics, charge-exchange processes, collective as well as singleparticle excitation and ionization, electron-electron correlation effects in atoms and in solids, excitation and single and multiple ionization of various targets, energy loss, scattering and channelling of primary particles and electron and photon emission processes.

The MPS conference series continues, and the next conference was planned for 2020 in Turku, Finland. However, due to the escalating coronavirus pandemic and the unforeseeable duration of the disruption of activities, it is postponed either to 2021 or 2022. Similarly to the previous MPS conferences, it will focus on the interaction of light and charged particles (ions and electrons) with various forms of matter, ranging from atoms to nanoparticles and surfaces. In particular the latest experiments, techniques and theoretical results involving multiple particles, their interaction (correlation, scattering, dynamics, multi-photon absorption) and detection (such as coincident experiments) are presented.

Acknowledgements The excellent cooperation of all the authors who have submitted papers to this Topical Issue, the reviewers who have taken time to analyse the submissions and the Editors and Publishers for bringing together these papers into a complete issue is greatly acknowledged. 


\section{Author contributions}

All the authors contributed equally in writing this Editorial.

Data Availability Statement This manuscript has no associated data or the data will not be deposited. [Authors' comments: This is an editorial for the Topical Issue on many particle spectroscopy of atoms, molecules, clusters and surfaces and all data generated are included into this published article.]

\section{References}

1. J. Berakdar, J. Kirschner (eds.), Many-Particle Spectroscopy of Atoms, Molecules, Clusters and Surfaces (Kluwer Academic, Plenum Publishers, New York, 2001)

2. M. Takahashi, K. Ueda (eds.), in International Conference on Many Particle Spectroscopy of Atoms, Molecules, Clusters and Surfaces, Tohoku University, Sendai, 2010, J. Phys.: Conf. Ser., Vol. 288 (2011)

3. A. Wobst, T. Lischke, B. Langer, A. Dorn, U. Becker, In International Conference on Many Particle Spectroscopy of Atoms (Clusters and Surfaces, Berlin, 2012)

4. L.U. Ancarani (ed.), in International Conference on Many Particle Spectroscopy of Atoms, Molecules, Clusters and Surfaces, Metz,, 2014, J. Phys.: Conf. Ser., Vol. $601(2015)$

5. A.N. Grum-Grzhimailo, Y.V. Popov, E.V. Gryzlova, A.V. Solov'yov, Eur. Phys. J. D 71, 201 (2017)

6. V. Borovik, V. Roman, A. Kupliauskiene, I. Shafranyosh, O. Borovik, Eur. Phys. J. D 73, 43 (2019)

7. M. Génévriez, P. Defrance, J.J. Jureta, X. Urbain, Eur. Phys. J. D 73, 30 (2019)

8. K. Tőkési, B. Paripás, E. Kovács, Eur. Phys. J. D 73, 84 (2019)

9. B. Paripás, B. Palásthy, M. Béres, Nucl. Instrum. Methods Phys. Res. B 369, 34 (2016)

10. M. Dampc, P. Możejko, M. Zubek, Eur. Phys. J. D 72, $216(2018)$

11. V. Oliveira, A. Herbert, A.C.F. Santos, K. Tőkési, Eur. Phys. J. D 73, 146 (2019)

12. A.C.F. Santos, W. Wolff, M.M. Sant'Anna, G.M. Sigaudand, R.D. DuBois, J. Phys. B: At. Mol. Opt. Phys. 46, $075202(2013)$

13. A.C.F. Santos, D.N. Vasconcelos, M.A. MacDonald, M.M. Sant'Anna, B.N.C. Tenório, A.B. Rocha, V. Morcelle, N. Appathurai, L. Zuin, Eur. Phys. J. D 73, 81 (2019)
14. J.B. Maljković, J. Vuković, K. Tőkési, B. Predojević, B.P. Marinković, Eur. Phys. J. D 73, 27 (2019)

15. M.A. Pocsai, I.F. Barna, K. Tőkési, Eur. Phys. J. D 73, $74(2019)$

16. S.D. Lopez, D.G. Arbo, Eur. Phys. J. D 73, 28 (2019)

17. N.M. Novikovskiy, D.V. Rezvan, N.M. Ivanov, I.D. Petrov, B.M. Lagutin, A. Knie, A. Ehresmann, PhV Demekhin, V.L. Sukhorukov, Eur. Phys. J. D 73, 22 (2019)

18. B.M. Lagutin, I.D. Petrov, N.M. Novikovskiy, V.L. Sukhorukov, PhV Demekhin, A. Knie, A. Ehresmann, Eur. Phys. J. D 73, 103 (2019)

19. E.V. Gryzlova, A.N. Grum-Grzhimailo, M.D. Kiselev, S.M. Burkov, Eur. Phys. J. D 73, 93 (2019)

20. Y. Orimo, K. Tőkési, T. Sato, K.L. Ishikawa, Eur. Phys. J. D 73, 153 (2019)

21. N.I. Shvetsov-Shilovski, M. Lein, K. Tőkési, Eur. Phys. J. D 73, 37 (2019)

22. N.M. Novikovskiy, D.V. Rezvan, I.D. Petrov, B.M. Lagutin, PhV Demekhin, V.L. Sukhorukov, Eur. Phys. J. D 73, 83 (2019)

23. N.M. Novikovskiy, V.L. Sukhorukov, A.N. Artemyev, PhV Demekhin, Eur. Phys. J. D 73, 79 (2019)

24. L. Budai, Z. Márton, P. Dombi, K. Tőkési, Eur. Phys. J. D 73, 138 (2019)

25. S.A. Zaytsev, A.S. Zaytsev, L.U. Ancarani, K.A. Kouzakov, Eur. Phys. J. D 73, 42 (2019)

26. A.S. Zaytsev, D.S. Zaytseva, L.U. Ancarani, S.A. Zaytsev, Eur. Phys. J. D 73, 111 (2019)

27. L.H. Yang, K. Tőkési, B. Da, Z.J. Ding, Eur. Phys. J. D 73, 21 (2019)

28. J.M. Gong, L.H. Yang, K. Tőkési, Z.J. Ding, Eur. Phys. J. D 73, 24 (2019)

29. E. Acebal, S. Otranto, Eur. Phys. J. D 73, 91 (2019)

30. T. Mukoyama, K. Tökési, Eur. Phys. J. D 73, 120 (2019)

31. T. Mukoyama, Eur. Phys. J. D 73, 75 (2019)

32. N. Bachi, S. Otranto, Eur. Phys. J. D 73, 4 (2019)

33. A.I. Bondarev, Y.S. Kozhedub, I.I. Tupitsyn, V.M. Shabaev, G. Plunien, Eur. Phys. J. D 73, 46 (2019)

34. S. Otranto, N. Bachi, R.E. Olson, Eur. Phys. J. D 73, $41(2019)$

35. O. Asztalos, B. Szondy, K. Tőkési, G.I. Pokol, Eur. Phys. J. D 73, 116 (2019)

36. G. Zhao, T. Miteva, N. Sisourat, Eur. Phys. J. D 73, 69 (2019)

37. Sz Hack, Z. Tóth, S. Varró, A. Czirják, Eur. Phys. J. D 73, 77 (2019)

38. Cavalieri et al., Lett. Nature 449, 1029 (2007)

39. C. Lemell et al., Phys. Rev. A 79, 062901 (2009)

40. S. Nagele et al., J. Phys. B 44, 081001 (2011)

41. E. Goulielmakis et al., Nature 466, 739 (2010)

42. R. Kienberger et al., Nature 427, 817 (2004)

43. C. Lemell et al., Phys. Rev. A 85, 011403 (2012) 